\title{
Prognostic Effects of Treatment Protocols for Febrile Convulsive Status Epilepticus in Children
}

\section{Shoichi Tokumoto ( $\nabla$ toku6012@med.kobe-u.ac.jp )}

Kobe University Graduate School of Medicine

\section{Masahiro Nishiyama}

Kobe University Graduate School of Medicine

Hiroshi Yamaguchi

Kobe University Graduate School of Medicine

Kazumi Tomioka

Kobe University Graduate School of Medicine

Yusuke Ishida

Hyogo Prefectural Kobe Children's Hospital

\section{Daisaku Toyoshima}

Hyogo Prefectural Kobe Children's Hospital

Hiroshi Kurosawa

Hyogo Prefectural Kobe Children's Hospital

Kandai Nozu

Kobe University Graduate School of Medicine

\section{Azusa Maruyama}

Hyogo Prefectural Kobe Children's Hospital

Ryojiro Tanaka

Hyogo Prefectural Kobe Children's Hospital

Kazumoto lijima

Kobe University Graduate School of Medicine

Hiroaki Nagase

Kobe University Graduate School of Medicine

\section{Research Article}

Keywords: febrile seizure, clinical protocol, anticonvulsant, barbiturate, benzodiazepine, phenytoin

Posted Date: October 28th, 2021

DOI: https://doi.org/10.21203/rs.3.rs-993441/v1 
License: (c) (i) This work is licensed under a Creative Commons Attribution 4.0 International License. Read Full License

Version of Record: A version of this preprint was published at BMC Neurology on March 5th, 2022. See the published version at https://doi.org/10.1186/s12883-022-02608-2. 


\section{Abstract}

Background: Febrile status epilepticus is the most common form of status epilepticus in children. No previous reports compare the effectiveness of treatment strategies using fosphenytoin (fPHT) or phenobarbital (PB) and those using anesthetics as second-line anti-seizure medication for benzodiazepine-resistant convulsive status epilepticus (CSE). We aimed to examine the outcomes of various treatment strategies for febrile convulsive status epilepticus (FCSE) in a real-world setting while comparing the effects of different treatment protocols and their presence or absence.

Methods: This was a single-center historical cohort study that was divided into three periods. Patients who presented with febrile convulsive status epilepticus for $\geq 60$ min even after the administration of at least one anticonvulsant were included. During period I (October 2002-December 2006), treatment was performed at the discretion of the attending physician, without a protocol. During period II (January 2007-February 2013), barbiturate coma therapy (BCT) was indicated for FCSE resistant to benzodiazepines. During period III (March 2013-April 2016), BCT was indicated for FCSE resistant to fPHT or PB.

Results: The rate of EEG monitoring was lower in period I than period II+III $(7.7 \%$ vs. $83.3 \%, p<0.01)$. Midazolam was administered by continuous infusion more often in period I than period II+III $(84.6 \%$ vs. $25.0 \%, p<0.01)$, whereas fPHT was administered less often in period I than period II+III ( $0 \%$ vs. $27.4 \%$, $\mathrm{p}<0.01)$. The rate of poor outcome, which was determined using the Pediatric Cerebral Performance Category scale, was higher in period I than period II+III $(23.1 \%$ vs. $7.1 \%, p=0.03)$. The rate of poor outcome did not differ between periods II and III ( $4.2 \%$ vs. $11.1 \%, \mathrm{p}=0.40)$.

Conclusions. While the presence of a treatment protocol for FCSE in children may improve outcomes, a treatment protocol using fPHT or PB may not be associated with better outcomes.

\section{Background}

Status epilepticus (SE) is one of the most common neurological emergencies in children, with an incidence of 18 to 41 cases per 100,000 children per year [1]. The mortality rate is $2.7-8 \%$, and the rate of sequelae (mainly neurological) is $10-20 \%$ [2]. Prolonged febrile convulsions are the most common etiology of SE in childhood [3]. Febrile status epilepticus (FSE) is the most common form of SE in children, which accounts for $25 \%$ of all childhood SE[4]. Regarding treatment, various guidelines have been published targeting convulsive status epilepticus (CSE) and these guidelines recommend benzodiazepines as the first treatment for CSE [5-8]. In cases of resistance to benzodiazepines, nonanesthetic anticonvulsants are the primary choice. For example, the American Epilepsy Society recommends fosphenytoin (fPHT), valproic acid, or levetiracetam as second-line therapies, and, if these agents are not available, it recommends phenobarbital (PB) [5]. There is no clear evidence on additional treatments when cases are resistant to these second-line therapies, but usually anesthetic therapies are used [5-7]. Although treatment protocols for CSE in children are currently being developed in many 
countries and communities, there have been no reports examining the effects of the presence or absence of such protocols on treatment outcomes. In addition, there are no reports comparing the effectiveness of treatment strategies using fPHT or PB and those using anesthetics as second-line anti-seizure medication for benzodiazepine-resistant CSE.

Therefore, we examined real-world comparative effects of different treatment strategies for febrile CSE (FCSE) on patient outcomes, focusing on two points as follows: (1) comparison of the effects of the presence or absence of a protocol, and (2) comparison of the effects of different protocols. Our main hypothesis was that the presence of a treatment protocol would improve outcomes in children with FCSE. In addition, we hypothesized that protocols using fPHT or PB would lead to better outcomes than those without $\mathrm{FPHT}$ or $\mathrm{PB}$, and that protocols using $\mathrm{PPHT}$ or $\mathrm{PB}$ would reduce the number of times anesthetic therapy is administered to children with FCSE.

\section{Methods}

\subsection{Study design}

This was a single-center historical cohort study that was divided into three periods. We used data gathered in the framework of a prospectively collected medical database of consecutive cases admitted to Kobe Children's Hospital, Japan, with convulsive seizures accompanied by fever, and reviewed related clinical charts.

\subsection{Definitions}

SE was defined as a continuous seizure that lasted for at least $5 \mathrm{~min}$, or a sequence of intermittent seizures without full recovery of consciousness between seizures [9]. CSE was defined as a seizure with overt motor symptoms longer than 30 min or recurrent seizures that lasted for a total of more than 30 min without the patient fully regaining consciousness [10]. FCSE was defined as a condition of febrile convulsive SE that persisted for 60 min or longer even after the administration of at least one anticonvulsant (i.e., benzodiazepine).

\subsection{Participants}

We extracted the records of pediatric patients (aged $<18$ years) admitted to Hyogo Children's Hospital for convulsive seizures with fever and met the definition of FCSE between October 2002 and April 2016. We excluded the records of children who had a history of neurological problems before each hospitalization. The records of children with multiple hospitalizations were also excluded except for the first hospitalization episode. Children diagnosed with encephalitis, such as acute disseminated encephalomyelitis [11], limbic encephalitis [12], and pleocytosis in the cerebrospinal fluid (> 8 cells $/ \mu L)$, were excluded. Children with multiple organ failure were also excluded.

\subsection{Procedures}


The time between October 2002 and April 2016 was divided into three periods (Figure 1). During period I (October 2002-December 2006), treatment was administered at the discretion of the attending physician, without a protocol. In practice, FCSE has often been treated with midazolam continuous infusion (MDL $\mathrm{ci})$, without electroencephalogram (EEG) monitoring. There were no common rules among physicians regarding the timing of therapeutic interventions in period I. During period II (January 2007-February 2013), barbiturate coma therapy (BCT) was indicated for FCSE that was resistant to first-line drugs (mainly benzodiazepines). The protocol which had been used in period II was revised because PB and fPHT became available in Japan. After that, during period III (March 2013-April 2016), BCT was indicated for FCSE resistant to second-line drugs (fPHT or PB). During period II and III, the protocol was decided based on a consensus among pediatric neurologists, acute care physicians, and intensivists. Thiamylal was mainly used for BCT. During period I, targeted temperature management (TTM) was sometimes implemented at the discretion of the attending physician. During periods II and III, FCSE was treated with TTM when BCT was indicated. TTM was performed in accordance with previous reports [13]. Briefly, TTM controls body temperature using a cooling blanket (target $34^{\circ} \mathrm{C}$ or $36^{\circ} \mathrm{C}$ ) with ventilation, muscle relaxant drugs, and vasopressors, as needed. Continuous EEG monitoring was also performed during BCT.

\subsection{Outcomes}

The primary outcome was determined using the Pediatric Cerebral Performance Category (PCPC) scale score at the time of the last medical examination, within 90 days after onset. PCPC scale score was determined by pediatric neurologists. A PCPC scale score $\geq 2$ was defined as a poor outcome. The PCPC scale is scored as follows: 1 , normal; 2 , mild disability; 3 , moderate disability; 4 , severe disability; 5 , coma or vegetative state; 6 , brain death [14]. The secondary outcomes were final diagnosis and ventilatorassociated pneumonia (VAP) as treatment complications. The final diagnosis of FCSE consisted of acute encephalopathy $(A E)$ or febrile seizure. The diagnosis of $A E$, such as $A E$ with biphasic seizures and late reduced diffusion (AESD), or clinically mild encephalitis/encephalopathy with a reversible splenial lesion, was based on published literature [15]. The diagnosis of VAP was made using guidelines of the Infectious Disease Society of America [16]. Period I and period II+III were compared to investigate the effects of the presence or absence of a treatment protocol. In addition, period II and period III were compared to investigate the effects of different protocols, with or without second-line drugs, on FCSE that was resistant to first-line drugs.

\subsection{Data analyses}

Results are expressed as number (\%) or median (interquartile range [ 1 st quartile, 3rd quartile]). The Mann-Whitney $U$ test or Fisher's exact test were used, when appropriate, for statistical analysis of the results. Statistical analyses were performed using EZR, version 1.41 (Saitama Medical Center, Jichi Medical University, Saitama, Japan). A p-value $<0.05$ was considered statistically significant.

\section{Results}


From October 2002 to April 2016, 774 patients were hospitalized due to convulsive seizures with fever (Figure 2). Of these, 190 patients met the definition of FCSE. From this group, 67 patients with a history of neurological disorders, 3 patients with multiple hospitalizations, 7 patients with encephalitis, and 3 patients with multiple organ failure were excluded. Ultimately, 110 patients were included in the study. Period I included 26 patients, period II comprised 48 patients, and period III consisted of 36 patients.

\subsection{Comparison of the effects of the presence or absence of a protocol (period I vs. period II+III)}

Demographic and clinical characteristics of patients are shown in Table 1. The median patient age was significantly lower in period I $(16.5[10,29.5]$ months) than in period II+III $(22[16,39.25]$ months $)$ $(p=0.03)$. The median convulsive seizure duration was $150(96.25,345)$ min during period I and 133.5 $(88.5,211.25)$ min during period II+III $(p=0.30)$. The number of patients who underwent EEG was significantly higher in period II+III than in period I $(p<0.01)$. The number of patients who underwent intubation was $15(57.7 \%)$ in period I and $41(48.8 \%)$ in period II+III $(p=0.504)$. Eleven $(42.3 \%)$ patients underwent TTM during period I and $38(45.2 \%)$ during period II+III $(p=0.83)$. Among the antiepileptic drugs used, MDL ci was used more often in period I, while fPHT was used more often in period II+III $(p<0.001)$.

The outcomes of these effects are shown in Table 2. The rate of poor outcomes (PCPC scale score $\geq 2$ ) was significantly higher in period I than in period II+III. The rate of $A E$ was significantly higher in period I than in period II+III. The rate of VAP was $0 \%(0$ out of 26$)$ in period I, and $17.1 \%$ (7 out of 84$)$ in period II + III $(p=0.20)$.

\subsection{Comparison of the effects of different protocols (period II vs. period III)}

The median convulsive seizure duration was $125(89.75,210)$ min during period II and $142.5(84,225.25)$ min during period III $(p=0.81)$. The number of patients who underwent EEG was similar in period II and period III $(p=0.38)$. The number of patients who underwent intubation was $23(47.9 \%)$ in period II and 18 $(50.0 \%)$ in period III $(\mathrm{p}=1)$. Twenty $(41.7 \%)$ patients underwent TTM during period II and $18(50.0 \%)$ during period III ( $p=0.51)$. Among the antiepileptic drugs, MDL ci was used more often in period II, and fPHT was used more often in period III.

The outcomes of this comparison are shown in Table 3. There were no differences between the two groups in the rate of poor outcomes (PCPC scale score $\geq 2$ ) or the rates of final diagnoses. The rate of VAP was $6.3 \%$ (3 out of 48 ) in period II and $11.1 \%$ ( 4 out of 36 ) in period III $(p=0.46)$.

\section{Discussion}


To the best of our knowledge, this is the first report comparing the neurological outcome before and after the implementation of treatment protocols for FCSE in children. This study suggests that treatment protocols for FCSE in children improve the outcomes. In contrast, treatment protocols using $\mathrm{FPHT}$ or PB were not associated with better outcomes nor avoidance of anesthetic therapy in children with FCSE.

The efficacy of second-line drugs such as $\mathrm{fPHT}$, valproate, and levetiracetam has been reported to be similar in patients with benzodiazepine-resistant SE [17]. Moreover, we have previously reported that there is no difference in the efficacy of $\mathrm{FPHT}$ and MDL ci in pediatric patients with benzodiazepineresistant SE [18]. Previous studies showed that the efficacy of several second-line agents was similar in patients with benzodiazepine-resistant SE, including febrile SE. However, the effects with or without treatment protocols, or those due to differences in protocols in a real-world setting are yet to be clarified.

\subsection{Comparison of the effects of the presence or absence of a protocol (period I vs period II+III)}

There are several reports on the usefulness of treatment protocols for SE in children [19, 20]. Xie et al. [19] reported that before and after the implementation of a protocol, the time between the first detection of seizures and the administration of first- and second-line drugs was significantly reduced. Cassel et al. [20] also reported that the time to second-line drug administration was significantly reduced by the implementation of a protocol. Herein, the median time from seizure onset to BCT initiation was similar between period I and period II+III. Nevertheless, during period I, there were few patients in whom BCT was started more than $24 \mathrm{~h}$ after seizure onset (range, 2.5-85.2 h). In period II + III, BCT was started within 24 $\mathrm{h}$ after onset in all patients (range, 2.1-21.3 h). In period I, BCT was started more than $24 \mathrm{~h}$ after onset in three patients, and the outcomes of two of these patients were poor. As reported in previous studies, we similarly found that treatment protocols made earlier treatment possible, which may have resulted in better outcomes for the patients in our study.

Although there was no standard protocol in period $\mathrm{I}$, in practice many patients were treated with MDL ci without EEG monitoring to achieve termination of clinical seizures. In a previous study, 164 patients with SE underwent continuous EEG monitoring, and $48 \%$ of the patients had persistent electrographic seizures, even after the apparent seizures were suppressed [21]. Nagase et al. [22] reported that five out of eight children who received MDL ci for refractory FSE had breakthrough seizures, and concluded that clinical seizure control using MDL ci without EEG monitoring is insufficient to prevent neurological damage. Our results might potentially be explained by the presence or absence of EEG monitoring.

The rate of final diagnosis of $A E$ was significantly higher in period I than in period II+III. Tighter control of seizures with EEG monitoring might have suppressed excitotoxicity and the development of AE. Indeed, prolonged seizures cause excitotoxicity [23], and the association of excitotoxicity with the pathophysiology of $A E$, such as AESD, has been reported [24, 25]. 
In the present study, the median patient age was lower in period I than in period II+III. In a systematic review of outcomes from pediatric CSE, younger patients had higher rates of sequelae and mortality [10]. The poorer outcomes of patients in period I than those of patients in period II+III in our study may be due to the younger age of the patients.

\subsection{Comparison of the effects of different protocols (period II vs period III)}

The outcomes were not different between treatment protocols with and without fPHT or PB. We expected better outcomes in period III than period II, and the use of second-line drugs to reduce the number of cases of BCT, resulting in a reduction of VAP, a complication of mechanical ventilation. However, the use of second-line drugs did not improve outcomes or reduce the VAP incidence.

In period II, the proportion of patients receiving MDL ci was higher than that in period III. However, contrary to treatment in period I, treatment in periods II and III was performed under EEG monitoring. This allowed breakthrough seizures to be detected, which might explain the lack of differences in outcome. Treatment using fPHT occurred more frequently in period III than in period II. As a second-line treatment for benzodiazepine-resistant SE, fPHT is used worldwide [5-8]. However, its effect on febrile seizures is still unknown, and some reports have shown negative effects. Olson et al. [26] investigated the preventive effects of PB, valproic acid, and phenytoin on hyperthermia-induced seizures in rat pups. Animals injected with PB and valproic acid had seizure temperature thresholds that were significantly higher than those of controls, while animals injected with phenytoin had seizure temperature thresholds that were equal to or lower than those of controls. Among 56 patients experiencing 62 episodes of childhood FSE, phenytoin administration terminated seizures in 9 (14.5\%) episodes and failed to terminate them in 25 (40.3\%) episodes [27]. Atypical febrile seizures, including SE, are reported to be associated with sodium channel mutations [28-30]. Phenytoin, a sodium channel blocker, may have little effect on FSE [27]. In our study, during period III, fPHT was used mainly as a second-line drug. The lack of difference in outcomes between periods II and III may be due to the poor effect of fPHT on FSE. In recent years, studies have reported that the therapeutic effect of levetiracetam on $S E$ is equivalent to that of phenytoin [31, 32]. Reductions in the severity and mortality of hyperthermic seizures have been observed in levetiracetamtreated rats with cortical dysplasia, where levetiracetam exerted protective effects against hyperthermic seizure-induced blood-brain barrier disruption [33]. In childhood FSE outcomes may be improved by using levetiracetam instead of phenytoin as a second-line drug.

Outcomes of SE are affected by pre-existing neurological abnormalities [34, 35], but a major strength of the present study is that it only targeted patients without pre-existing neurological abnormalities. However, this study also has some limitations. First, it was an observational study without controls, based at a single center. Second, although the data were extracted from a prospectively collected database, the study itself was retrospective. It is unlikely that all of the listed exclusion criteria were documented accurately in every patient. Third, this study targeted febrile seizures, not afebrile seizures 
such as epilepsy and hypoxic-ischemic encephalopathy. Therefore, the results are not applicable to seizures caused by other factors.

\section{Conclusions}

Our study showes that the presence of a treatment protocol for FCSE in children may improve patient outcomes. Furthermore, our results suggest that a treatment protocol that includes the use of fPHT or PB may not be associated with better outcomes or help to elude the need for anesthetic therapy in these children.

\section{Abbreviations}

SE: Status epilepticus

CSE: convulsive status epilepticus

FSE: Febrile status epilepticus

FCSE: febrile convulsive status epilepticus

fPHT: fosphenytoin

PB: phenobarbital

MDL ci: midazolam continuous infusion

BCT: barbiturate coma therapy

TTM: targeted temperature management

EEG: electroencephalogram

PCPC: Pediatric Cerebral Performance Category

VAP: ventilator-associated pneumonia

AE: acute encephalopathy

AESD: acute encephalopathy with biphasic seizures and late reduced diffusion

\section{Declarations}

\section{Ethics approval and consent to participate}


All methods were carried out in accordance with the Declaration of Helsinki. The study design was approved by the ethics committee of Kobe Children's Hospital and the Kobe University Graduate School of Medicine (No. 170019). The requirement of informed consent was waived because of the retrospective study design.

\section{Consent for publication}

Not applicable.

\section{Availability of data and materials}

The data sets in this study are available from the corresponding author on reasonable request.

\section{Competing interests}

The authors have no financial relationships relevant to this article to disclose. Potential conflicts of interest: Kazumoto lijima has received grants from Eisai Co., Ltd, and consulting fees from Takeda $P$ Pharma Co., Ltd.; Kandai Nozu has received consulting fees from Eisai Co., Ltd, and lecture fees from Sumitomo Dainippon Pharma Co., Ltd.

\section{Funding}

This work was supported in part by a Grant-in-Aid for Young Scientists (B) (18K15711) of JSPS KAKENHI and a Grant-in-Aid for Research on Measures of Intractable Diseases (H30-Nanji-Ippan-007) from the Japanese Ministry of Health, Labor and Welfare.

\section{Authors' contributions}

ST designed the project, participated in the data analysis and first drafted the manuscript. MN and HN designed and supervised the project and critically reviewed and revised the manuscript for important intellectual content. HY and KT participated in the data cleaning, supported in the data analysis and revised the manuscript for important intellectual content. YI, DT and AM supported in the analysis and interpretation and revised the manuscript for important intellectual content. HK, KN and RT participated in the data analysis and interpretation of results, and revised the manuscript for important intellectual content. KI contributed to data analysis and interpretation, critical revision of the article and final approval of the version to be published. All authors approved the final manuscript as submitted and agree to be accountable for all aspects of the work. The corresponding author attests that all listed authors meet authorship criteria and that no others meeting the criteria have been omitted.

\section{Acknowledgements}

We thank Drs. Yusuke Seino, Kazunori Aoki, Naoko Tanizawa, Satoshi Matsui, Shinsuke Kajihara, Mayumi Kusumoto, Takuro Hayashi, Yoshimichi Yamaguchi, Hiroki Takeda, and other physicians in the Division of Pediatric Critical Care Medicine and the Department of Emergency and General Pediatrics of 
Hyogo Prefectural Kobe Children's Hospital for treating these patients. In addition, we thank the Clinical and Translational Research Center of Kobe University for the statistical analysis. We also thank Editage (www.editage.com) for the English language editing.

\section{References}

1. Lambrechtsen FA and Buchhalter JR Aborted and refractory status epilepticus in children: a comparative analysis. Epilepsia. 2008;49:615-25.

2. Sofou K, Kristjansdottir R, Papachatzakis NE, Ahmadzadeh A, Uvebrant P. Management of prolonged seizures and status epilepticus in childhood: a systematic review. J Child Neurol. 2009;24:918-26.

3. Singh RK, Gaillard WD. Status epilepticus in children. Curr Neurol Neurosci Rep. 2009;9:137-44.

4. Seinfeld S, Shinnar S, Sun S, Hesdorffer DC, Deng X, Shinnar RC, et al. Emergency management of febrile status epilepticus: results of the FEBSTAT study. Epilepsia. 2014;55:388-95.

5. Glauser T, Shinnar S, Gloss D, Alldredge B, Arya R, Bainbridge J, et al. Evidence-based Guideline: Treatment of convulsive status epilepticus in children and adults: Report of the Guideline Committee of the American Epilepsy Society. Epilepsy Curr. 2016;16:48-61.

6. Capovilla G, Beccaria F, Beghi E, Minicucci F, Sartori S, Vecchi M. Treatment of convulsive status epilepticus in childhood: recommendations of the Italian League Against Epilepsy. Epilepsia. 2013;54:23-34.

7. Friedman J. Emergency management of the paediatric patient with generalized convulsive status epilepticus. Paediatr Child Health. 2011;16:91-104.

8. National Institute for Health and Care Excellence. Epilepsies: diagnosis and management. London: National Institute for Health and Care Excellence. Available from: https://www.nice.org.uk/guidance/cg137. Accessed [cited 2021 October 17]

9. Lowenstein DH, Alldredge BK. Status epilepticus. N Engl J Med. 1998;338:970-6.

10. Raspall-Chaure M, Chin RFM, Neville BG, Scott RC. Outcome of paediatric convulsive status epilepticus: a systematic review. Lancet Neurol. 2006;5:769-79.

11. Krupp LB, Banwell B, Tenembaum S, International Pediatrics MS Study Group. Consensus definitions proposed for pediatric multiple sclerosis and related disorders. Neurology. 2007;68:S7-12.

12. Asztely F, Kumlien E. The diagnosis and treatment of limbic encephalitis. Acta Neurol Scand. 2012;126:365-75.

13. Nishiyama M, Tanaka T, Fujita K, Maruyama A, Nagase H. Targeted temperature management of acute encephalopathy without AST elevation. Brain Dev. 2015;37:328-33.

14. Fiser DH. Assessing the outcome of pediatric intensive care. J Pediatr. 1992;121:68-74.

15. Mizuguchi M, Ichiyama T, Imataka G, Okumura A, Goto T, Sakuma H, et al. Guidelines for the diagnosis and treatment of acute encephalopathy in childhood. Brain Dev. 2021;43:2-31.

16. Kalil AC, Metersky ML, Klompas M, Muscedere J, Sweeney DA, Palmer LB, at al. Management of adults with hospital-acquired and ventilator-associated pneumonia: 2016 Clinical Practice Guidelines 
by the Infectious Diseases Society of America and the American Thoracic Society. Clin Infect Dis. 2016;63:e61-111.

17. Chamberlain JM, Kapur J, Shinnar S, Elm J, Holsti M, Babcock L, at al Efficacy of levetiracetam, fosphenytoin, and valproate for established status epilepticus by age group (ESETT): a double-blind, responsive-adaptive, randomised controlled trial. Lancet. 2020;395:1217-24.

18. Nishiyama M, Nagase $H$, Tomioka $K$, Tanaka T, Yamaguchi $H$, Ishida $Y$, et al. Fosphenytoin vs. continuous midazolam for pediatric febrile status epilepticus. Brain Dev. 2018;40:884-90.

19. Xie Y, Morgan R, Schiff L, Hannah D, Wheless J, et al A computerized standard protocol order entry for pediatric inpatient acute seizure emergencies reduces time to treatment. J Child Neurol. 2014;29:162-6.

20. Cassel-Choudhury G, Beal J, Longani N, Leone B, Rivera R, Katyal C. Protocol-driven management of convulsive status epilepticus at a tertiary children's hospital: a quality Improvement Initiative. Pediatr Crit Care Med. 2019;20:47-53.

21. DeLorenzo RJ, Waterhouse EJ, Towne AR, Boggs JG, Ko D, DeLorenzo GA, et al. Persistent nonconvulsive status epilepticus after the control of convulsive status epilepticus. Epilepsia. 1998;39:833-40.

22. Nagase H, Nishiyama M, Nakagawa T, Fujita K, Saji Y, Maruyama A. Midazolam fails to prevent neurological damage in children with convulsive refractory febrile status epilepticus. Pediatr Neurol. 2014;51:78-84.

23. Olney JW, Collins RC, Sloviter RS. Excitotoxic mechanism of epileptic brain damage. Adv Neurol. 1986;44:857-77.

24. Takanashi J, Tada H, Terada H, Barkovich AJ. Excitotoxicity in acute encephalopathy with biphasic seizures and late reduced diffusion. AJNR Am J Neuroradiol. 2009;30:132-5.

25. Takanashi JI, Murofushi Y, Hirai N, Sano K, Matsuo E, Saito K, et al. Prognostic value of MR spectroscopy in patients with acute excitotoxic encephalopathy. J Neurol Sci. 2020;408:116636.

26. Olson JE, Scher MS, Holtzman D. Effects of anticonvulsants on hyperthermia-induced seizures in the rat pup. Epilepsia. 1984;25:96-9.

27. Ismail S, Levy A, Tikkanen H, Severe M, Wolters FJ, Carmant L. Lack of efficacy of phenytoin in children presenting with febrile status epilepticus. Am J Emerg Med. 2012;30:2000-4.

28. Nakayama J. Progress in searching for the febrile seizure susceptibility genes. Brain Dev. 2009;31:359-65.

29. Saitoh $M$, Ishii A, Ihara $Y$, Hoshino A, Terashima $H$, Kubota $M$, et al. Missense mutations in sodium channel SCN1A and SCN2A predispose children to encephalopathy with severe febrile seizures. Epilepsy Res. 2015;117:1-6.

30. Lucas PT, Meadows LS, Nicholls J, Ragsdale DS. An epilepsy mutation in the beta1 subunit of the voltage-gated sodium channel results in reduced channel sensitivity to phenytoin. Epilepsy Res. 2005;64:77-84. 
31. Dalziel SR, Borland ML, Furyk J, Bonisch M, Neutze J, Donath S, et al. Levetiracetam versus phenytoin for second-line treatment of convulsive status epilepticus in children (ConSEPT): an openlabel, multicentre, randomised controlled trial. Lancet. 2019;393:2135-45.

32. Lyttle MD, Rainford NEA, Gamble C, Messahel S, Humphreys A, Hickey H, et al. Levetiracetam versus phenytoin for second-line treatment of paediatric convulsive status epilepticus (EcLiPSE): a multicentre, open-label, randomised trial. Lancet. 2019;393:2125-34.

33. Ahishali B, Kaya M, Orhan N, Arican N, Ekizoglu O, Elmas I, et al. Effects of levetiracetam on bloodbrain barrier disturbances following hyperthermia-induced seizures in rats with cortical dysplasia. Life Sci. 2010;87:609-19.

34. Novorol CL, Chin RF, Scott RC. Outcome of convulsive status epilepticus: a review. Arch Dis Child. 2007;92:948-51.

35. Chin RFM, Neville BGR, Peckham C, Bedford H, Wade A, Scott RC. Incidence, cause, and short-term outcome of convulsive status epilepticus in childhood: prospective population-based study. Lancet. 2006;368:222-9.

\section{Tables}

Table 1. Demographic and clinical characteristics of patients by period 


\begin{tabular}{|c|c|c|c|c|c|c|}
\hline & $\begin{array}{l}\text { Period } \mathrm{Q} \\
\mathrm{n}=26\end{array}$ & $\begin{array}{l}\text { Period } \\
\text { II+III } \\
n=84\end{array}$ & $\begin{array}{l}p \\
\text { value }\end{array}$ & $\begin{array}{l}\text { Period II } \\
\mathrm{n}=48\end{array}$ & $\begin{array}{l}\text { Period III } \\
n=36\end{array}$ & $\begin{array}{l}\mathrm{p} \\
\text { value }\end{array}$ \\
\hline Sex, male, n (\%) & $13(50.0)$ & $38(45.2)$ & 0.66 & $\begin{array}{l}23 \\
(47.9)\end{array}$ & $\begin{array}{l}15 \\
(41.7)\end{array}$ & 0.82 \\
\hline Age, months & $\begin{array}{l}16.5(10 \\
29.5)\end{array}$ & $\begin{array}{l}22(16, \\
39.25)\end{array}$ & 0.03 & $\begin{array}{l}24(15 \\
47.5)\end{array}$ & $\begin{array}{l}20.5(16, \\
35)\end{array}$ & 0.45 \\
\hline History of febrile seizure, n (\%) & $3(11.5)$ & $21(25.0)$ & 0.18 & $\begin{array}{l}11 \\
(22.9)\end{array}$ & $\begin{array}{l}10 \\
(27.8)\end{array}$ & 0.62 \\
\hline Temperature on admission, ${ }^{\circ} \mathrm{C}$ & $\begin{array}{l}39.1 \\
(38.4 \\
39.8)\end{array}$ & $\begin{array}{l}38.65 \\
(38.1 \\
39.4)\end{array}$ & 0.10 & $\begin{array}{l}38.65 \\
(38.1 \\
39.4)^{\prime}\end{array}$ & $\begin{array}{l}38.65 \\
(38.1 \\
39.4)\end{array}$ & 0.93 \\
\hline Convulsive seizure duration & $\begin{array}{l}150 \\
(96.3 \\
345)\end{array}$ & $\begin{array}{l}133.5 \\
(88.5, \\
211.3)\end{array}$ & 0.30 & $\begin{array}{l}125 \\
(89.75 \\
210)\end{array}$ & $\begin{array}{l}142.5 \\
(84, \\
225.3)\end{array}$ & 0.81 \\
\hline Duration of hospital stay, days & $\begin{array}{l}9.5 \\
(4.75 \\
19.8)\end{array}$ & $7.5(5,13)$ & 0.17 & $\begin{array}{l}7(4 \\
12.3)\end{array}$ & $9(5,13)$ & 0.21 \\
\hline $\begin{array}{l}\text { Period from onset to } \\
\text { determination of outcome, } \\
\text { days }\end{array}$ & $\begin{array}{l}26(20 \\
39.8)\end{array}$ & $\begin{array}{l}32(27 \\
38.3)\end{array}$ & 0.70 & $\begin{array}{l}31(17.8 \\
37.8)\end{array}$ & $\begin{array}{l}35.5(30 \\
38.3)\end{array}$ & 0.06 \\
\hline EEG monitoring, n (\%) & $2(7.7)$ & $70(83.3)$ & $<0.001$ & $\begin{array}{l}38 \\
(79.2 \%)\end{array}$ & $\begin{array}{l}32 \\
(88.9 \%)\end{array}$ & 0.38 \\
\hline Intubation, n (\%) & $15(57.7)$ & $41(48.8)$ & 0.50 & $\begin{array}{l}23 \\
(47.9)\end{array}$ & $\begin{array}{l}18 \\
(50.0)\end{array}$ & 1 \\
\hline TTM, n (\%) & $11(42.3)$ & $38(45.2)$ & 0.83 & $\begin{array}{l}20 \\
(41.7)\end{array}$ & $\begin{array}{l}18 \\
(50.0)\end{array}$ & 0.51 \\
\hline Vasopressor, n (\%) & $13(50.0)$ & $37(44.0)$ & 0.66 & $\begin{array}{l}20 \\
(41.7)\end{array}$ & $\begin{array}{l}17 \\
(47.2)\end{array}$ & 0.66 \\
\hline $\begin{array}{l}\text { Number of antiepileptic drugs } \\
\text { used }\end{array}$ & $3(2,3)$ & $2(2,3)$ & 0.43 & $2(2,3)$ & $3(2,4)$ & 0.07 \\
\hline \multicolumn{7}{|l|}{$\begin{array}{l}\text { Details of antiepileptic drugs } \\
\text { used }\end{array}$} \\
\hline DZP, n (\%) & $25(96.2)$ & $74(88.1)$ & 0.45 & $\begin{array}{l}44 \\
(91.7)\end{array}$ & $\begin{array}{l}30 \\
(83.3)\end{array}$ & 0.31 \\
\hline MDL, n (\%) & $19(73.1)$ & $69(82.1)$ & 0.40 & $\begin{array}{l}38 \\
(79.2)\end{array}$ & $\begin{array}{l}31 \\
(86.1)\end{array}$ & 0.57 \\
\hline fPHT iv, n (\%) & $0(0)$ & $23(27.4)$ & 0.002 & $3(6.3)$ & $\begin{array}{l}20 \\
(55.6)\end{array}$ & $<0.001$ \\
\hline \multirow[t]{2}{*}{ PB, n (\%) } & $9(34.6)$ & 15 (17.9) & 0.10 & $\begin{array}{l}10 \\
(20.8)\end{array}$ & 5 (13.9) & 0.57 \\
\hline & & Page $14 / 18$ & & & & \\
\hline
\end{tabular}




\begin{tabular}{|c|c|c|c|c|c|c|}
\hline MDL ci, n (\%) & $22(84.6)$ & $21(25.0)$ & $<0.001$ & $\begin{array}{l}19 \\
(39.6)\end{array}$ & $2(5.6)$ & $<0.001$ \\
\hline Thiamylal ci, n (\%) & $9(34.6)$ & $38(45.2)$ & 0.37 & $\begin{array}{l}20 \\
(41.7)\end{array}$ & $\begin{array}{l}18 \\
(50.0)\end{array}$ & 0.51 \\
\hline $\begin{array}{l}\text { Time from onset to BCT } \\
\text { initiation, } h\end{array}$ & $\begin{array}{l}5.5(3.9, \\
37.1)\end{array}$ & $\begin{array}{l}6.8(5.0 \\
9.0)\end{array}$ & 0.97 & $\begin{array}{l}6.3(5.3, \\
9.0))\end{array}$ & $\begin{array}{l}6.9(4.9, \\
8.9)\end{array}$ & 0.87 \\
\hline
\end{tabular}

Data are shown as the median (interquartile range) unless otherwise noted.

EEG: electroencephalogram, TTM: targeted temperature management, DZP: diazepam, MDL: midazolam, fPHT: fosphenytoin, PB: phenobarbital, BCT: barbiturate coma therapy, iv: intravenous, ci: continuous infusion.

Table 2. Outcome comparison, period I vs. period II+III

\begin{tabular}{|c|c|c|c|}
\hline & $\begin{array}{l}\text { Period I } \\
n=26\end{array}$ & $\begin{array}{l}\text { Period II+III } \\
n=84\end{array}$ & $\mathrm{p}$ value \\
\hline PCPC scale score $\geq 2, n(\%)$ & $6(23.1)$ & $6(7.1)$ & 0.03 \\
\hline
\end{tabular}

\begin{tabular}{llll}
\hline Final diagnosis & & & \\
\hline AE, $\mathrm{n}(\%)$ & $9(34.6)$ & $11(13.1)$ & 0.02 \\
\hline AESD, $\mathrm{n}(\%)$ & $5(19.2)$ & $6(7.1)$ & 0.12 \\
\hline MERS, $\mathrm{n}(\%)$ & $0(0)$ & $1(1.2)$ & 1 \\
\hline Unclassified AE, n (\%) & $4(15.4)$ & $4(4.8)$ & 0.09 \\
\hline Febrile seizure, n (\%) & $17(65.4)$ & $73(86.9)$ & 0.02 \\
\hline Treatment-related complication & & & \\
\hline VAP, n (\%) & $0(0)$ & $7(8.3)$ & 0.20
\end{tabular}

PCPC: Pediatric Cerebral Performance Category, AE: acute encephalopathy, AESD: acute encephalopathy with biphasic seizures and late reduced diffusion, MERS: clinically mild encephalitis/ encephalopathy with a reversible splenial lesion, VAP: ventilator-associated pneumonia.

Table 3. Outcome comparison, period II vs. period III 


\begin{tabular}{llll} 
& $\begin{array}{l}\text { Period II } \\
\mathrm{n}=48\end{array}$ & $\begin{array}{l}\text { Period III } \\
\mathrm{n}=36\end{array}$ & $\mathrm{p}$ value \\
\hline PCPC scale score $\geq 2, \mathrm{n}(\%)$ & $2(4.2)$ & $4(11.1)$ & 0.40 \\
& & & \\
\hline Final diagnosis & & & \\
\hline AE, $\mathrm{n}(\%)$ & $7(14.6)$ & $4(11.1)$ & 0.75 \\
\hline AESD, $\mathrm{n}(\%)$ & $3(6.2)$ & $3(8.3)$ & 1 \\
\hline MERS, $\mathrm{n}(\%)$ & $1(2.1)$ & $0(0)$ & 1 \\
\hline Unclassified AE, $\mathrm{n}(\%)$ & $3(6.3)$ & $1(2.8)$ & 0.63 \\
\hline Febrile seizure, $\mathrm{n}(\%)$ & $41(85.4)$ & $32(88.9)$ & 0.75 \\
\hline Treatment-related complication & & & \\
\hline VAP, $\mathrm{n}(\%)$ & $3(6.3)$ & $4(11.1)$ & 0.46
\end{tabular}

PCPC: Pediatric Cerebral Performance Category, AE: acute encephalopathy, AESD: acute encephalopathy with biphasic seizures and late reduced diffusion, MERS: clinically mild encephalitis/ encephalopathy with a reversible splenial lesion, VAP: ventilator-associated pneumonia.

\section{Figures}



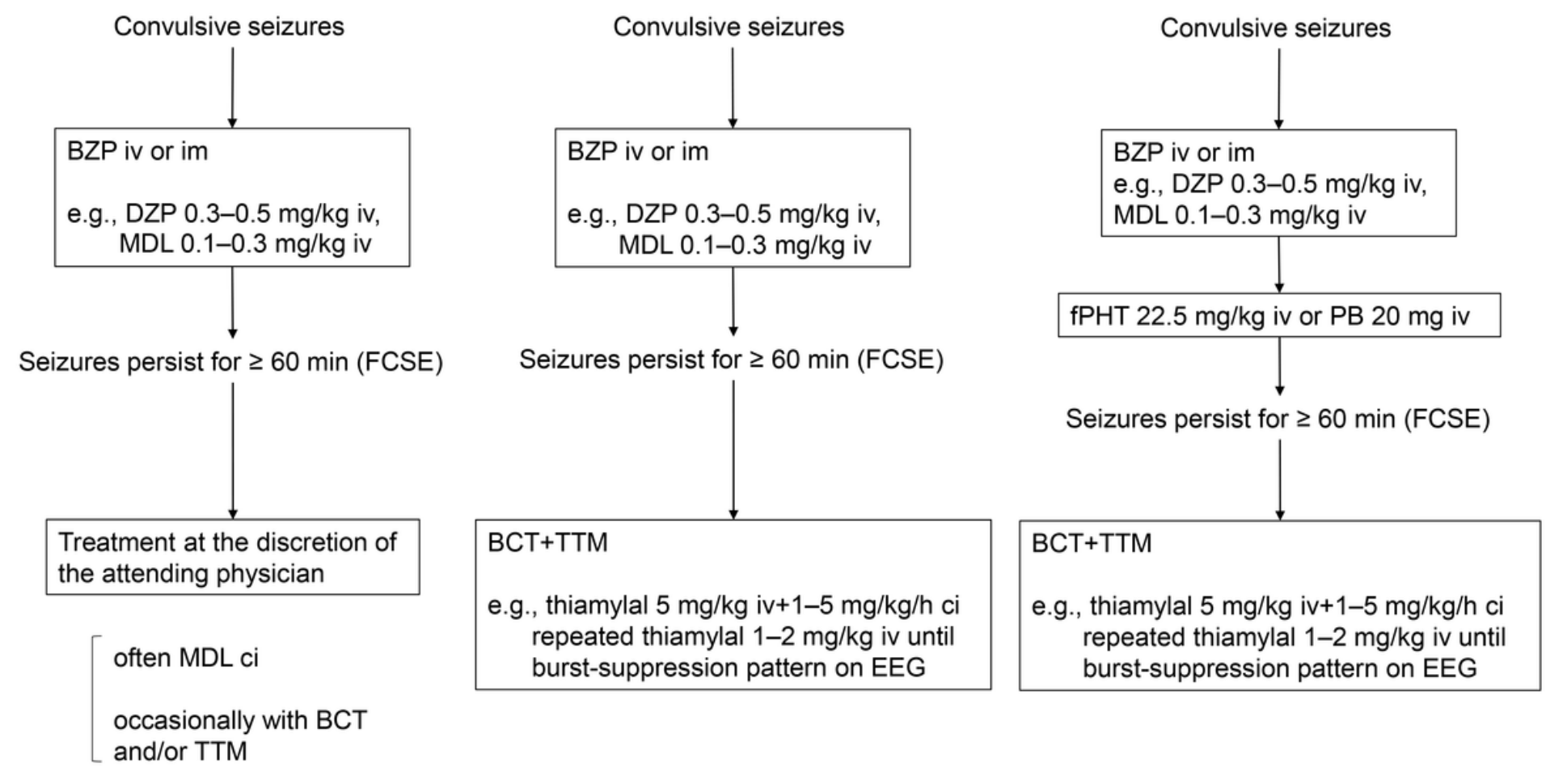

Period I

Period II

Period III

\section{Figure 1}

Treatment protocol for each period. BZP: benzodiazepine, DZP: diazepam, MDL: midazolam, FCSE: febrile convulsive status epilepticus, $\mathrm{FPHT}$ : fosphenytoin, PB: phenobarbital, BCT: barbiturate coma therapy, TTM: targeted temperature management, iv: intravenous, im: intramuscular, ci: continuous infusion, EEG: electroencephalogram. 
774 patients were admitted to Kobe Children's Hospital due to convulsive seizures with fever between October 2002 and April 2016

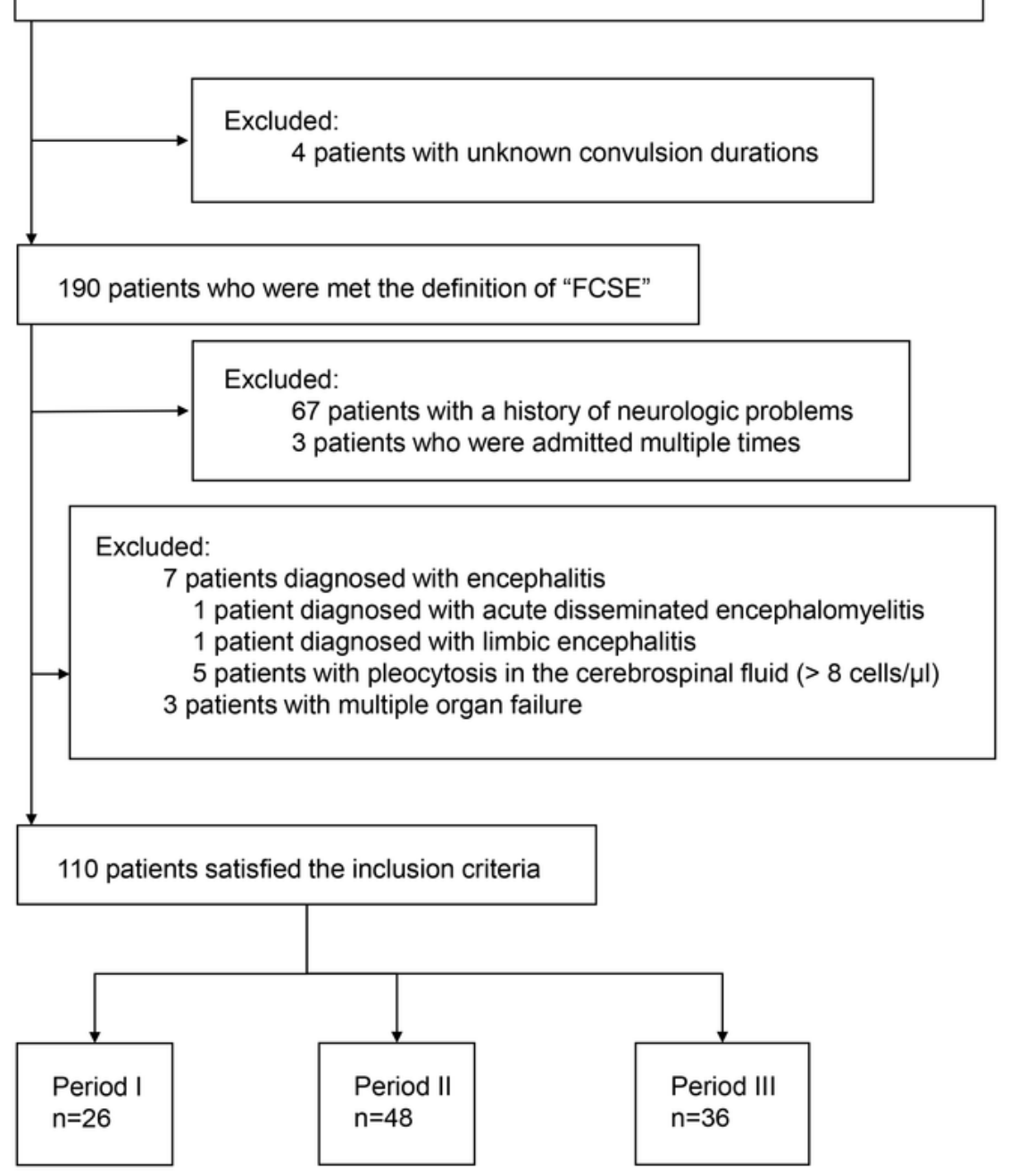

\section{Figure 2}

Selection of patients. The flowchart details the patient selection procedure. FCSE: febrile convulsive status epilepticus. 